\title{
A Reemergent Case of Bubonic Plague-Inner Mongolia Autonomous Region, China, July, 2020
}

\author{
Xiaona Shen ${ }^{1, \alpha} ;$ Jianyun $\mathrm{Li}^{2, \alpha} ;$ Mengguang Fan ${ }^{2} ;$ Lianxu Xia ${ }^{1}$; Wei Li ${ }^{1, *}$
}

On July 5, 2020, a confirmed case of bubonic plague was reported in Urat Middle Banner of Inner Mongolia Autonomous Region, China, which belongs to the Meriones unguiculatus plague focus in the Inner Mongolia Plateau. This was the same natural plague focus that reported 2 pneumonic plague patients and 2 bubonic plague patients in Inner Mongolia in late 2019 (1). The 2 pneumonic plague patients were once transferred into Beijing Municipality in November 2019 for comprehensive treatment; however, one patient still died.

The case of bubonic plague occurred in Inner Mongolia in a 47-year-old female shepherd that experienced fever, chills, and some symptoms of physical discomfort on July 2. On July 3, she sought treatment in the Urat Middle Banner People's Hospital because of high body temperature $\left(40{ }^{\circ} \mathrm{C}\right)$ with the swollen and painful right inguinal lymph nodes. A polymerase chain reaction (PCR) test for Yersinia pestis specific genes (cafl and YPO0392 gene) was performed in the Urat Middle Banner (county-level) CDC, and positive results were detected in the lymph node aspirate. Subsequently, the patient was suspected of having a case of bubonic plague and notified to the local public health agency on the same day. On July 4, Inner Mongolia Autonomous Region CDC reviewed the PCR test and the reverse indirect hemagglutination assay (RIHA) test. The lymph node aspirate sample was positive for the PCR and RIHA tests, while specimens of blood and pharyngeal swab were negative. According to the "plague diagnostic criteria" of China, the case was identified as a confirmed case of bubonic plague. As of July 10, 2020, the patient is being treated in a local general hospital and her condition is stable.

When the bubonic plague patient was initially identified as a suspected case, a series of prevention and control measures were adopted by the local government including the medical observation of 15 close contacts, the establishment of a isolation zone to prevent other residents from entering the patient's home and the surrounding neighborhood areas, and the implementation of rodent and flea control measures in the corresponding epizootic focus. As of July 10, no additional cases were reported.

As early as March 2020, animal plagues had been detected in the Urat Middle Banner with a total of eight Yersinia pestis strains having been isolated from local reservoirs (2). In June, four dead $M$. unguiculatus rodents were found in the region neighboring where the patient lived, and four $Y$. pestis strains were isolated from these dead hosts. This observation indicated that serious $M$. unguiculatus plague epizootics existed in that area. Epidemiological investigations suggested that the patient might have been infected from a flea bite that transmitted the disease through local epizootic rodents. This was suspected because the patient was a shepherd and suffered right inguinal bubonic plague when herding sheep on an $M$. unguiculatus plague natural focus, where Nosopsyllus laeviceps kuzenkovi is the main vector of plague (3).

Continuous animal plague have occurred in previous years (in 2018 and 2019) in the Meriones unguiculatus plague focus of Inner Mongolia, and in 2020, a total of 14 counties of 5 leagues (cities) had found animal plague in Inner Mongolia with 69 positive rodents been detected (2). These surveillance results indicated that animal plague epizootics in the $M$. unguiculatus plague focus were still active.

In the Meriones unguiculatus plague focus of the Inner Mongolian Plateau, most cases in humans were caused by flea bites and characterized as bubonic plague. Therefore, a key measure for human plague prevention and control was to carry out health education about rodent and flea control and to avoid flea bites in the epizootic areas. In addition, local residents needed to be educated to avoid hunting in or being in contact with wild reservoirs of plague.

Acknowledgments: We thank the colleagues that participated in the outbreak investigation, and we thank those who collected the surveillance data from the Inner Mongolia Autonomous Region.

doi: $10.46234 / \mathrm{ccdcw} 2020.145$

\# Corresponding author: Wei Li, liwei@icdc.cn. 
${ }^{1}$ State Key Laboratory of Infectious Disease Prevention and Control, National Institute for Communicable Disease Control and Prevention, Chinese Center for Disease Control and Prevention, Beijing, China;

${ }^{2}$ General Center for Disease Control and Prevention of Inner Mongolia Autonomous Region, Hohhot, Mongolia Autonomous Region, China.

\& Joint first authors.

Submitted: July 09, 2020; Accepted: July 10, 2020

\section{REFERENCES}

1. Wang YM, Zhou L, Fan MG, Wang QY, Li JY, Li Q, et al. Isolated Cases of Plague - Inner Mongolia-Beijing, 2019. China CDC Weekly 2019;1(1):13 - 6. http://dx.doi.org/10.46234/ccdcw2019.005.

2. Information Management System for Plague Control, China Information System for Disease Control and Prevention. Inner network. https://10.249.6.18:8881/cdc. [2020-7-9].

3. Liu J, Zhang W. Inner Mongolia Plague. Huhhot: Inner-Mongol People Publishing House. 1997. (In Chinese). 